Pacific Journal of Mathematics

EFFLUENT AND NONEFFLUENT FIXED POINTS ON 


\title{
EFFLUENT AND NONEFFLUENT FIXED POINTS ON DENDRITES
}

\author{
Helga Schirmer
}

\begin{abstract}
This paper gives a partial answer to the problem of establishing conditions for the existence of selfmaps of one-dimensional spaces with prescribed fixed points and fixed point indices. Two types of isolated fixed points on dendrites are defined, and called effiuent and noneffiuent fixed points. They correspond on polyhedral trees to fixed points of minimal or maximal algebraic index, but are characterized by separation properties. Necessary and sufficient conditions are given for the existence of a selfmap of a dendrite which has a prescribed set of effiuent and nonefiluent fixed points.
\end{abstract}

1. Introduction. It is known that dendrites, as well as many polyhedra, have the "complete invariance property" (see e.g. [7], [12]), which means that any arbitrarily given closed and nonempty subset of these spaces can be the fixed point set of a suitable selfmap. For a fairly large class of polyhedra this result has been sharpened considerably: necessary and sufficient conditions have been established for the existence of a selfmap within a given homotopy class for which not only the locations of the (finitely many) fixed points, but also their indices are prescribed [8]. The construction of such a selfmap uses Nielsen's theory of fixed point classes and the "splitting" and "moving" of fixed points. These methods fail completely in one-dimensional spaces and hence cannot be used for dendrites. Nevertheless the question arises under which conditions selfmaps of dendrites exist for which not only the locations of the fixed points, but also their indices are given.

We give here a partial answer to this question. Dendrites are acyclic continua, and hence our proofs employ methods which belong to continua theory. They consist of an exploitation of the connectedness properties of dendrites which can e.g. be found in [4] and [13], as well as of the partial order structure of dendrites which was developed by $L$. E. Ward, Jr. [9], [10] and has often been found useful in the investigation of fixed point questions. These methods belong to general topology, and it is therefore necessary to replace the usual definition of the fixed point index by a topological rather than an algebraic one. This is, however, done only for fixed points which correspond to those with a minimal or a maximal index. If $c$ is an isolated fixed point on a polyhedral tree and is of order $o(c)$, then one can easily check that its index $i(c)$ under different 
selfmaps can assume values for which $1-o(c) \leqq i(c) \leqq 1$. The so-called effluent fixed points defined in $\$ 2$ correspond to fixed points with index $1-o(c)$, and the noneffluent ones to those of index one. Inessential fixed points are those which correspond to fixed points of index zero. We do not consider fixed points which correspond to those of an index different from 1,0 , or $1-o(c)$.

Before we investigate the existence of selfmaps on dendrites with given effluent and noneffluent fixed points, we establish some properties of these types of fixed points. We show that noneffluent fixed points are always essential (Theorem 3.2), and that every selfmap of a dendrite has at least one noneffluent fixed point (Theorem 3.5). An effluent fixed point is essential if and only if it is not an endpoint (Theorem 4.1). A map need not have an effluent fixed point, and the existence of essential effluent fixed points implies the existence of more than one noneffluent one. Two lower bounds for the number of noneffluent fixed points in the presence of effluent fixed points are established in Theorems 4.2 and 4.4.

These properties are used in the proof of the main result (Theorem 5.2), which gives necessary and sufficient conditions for the existence of a selfmap of a dendrite which has a prescribed finite set of effluent and noneffluent fixed points. These conditions are more complicated than in the polyhedral case [8], and the case where all possible values of fixed point indices for selfmaps of dendrites are considered will likely turn out to be quite cumbersome.

A convex metric of the dendrite is used throughout. I do not know how far the results can be extended to trees, i.e., to the nonmetric case. The metric is not used in the definition of effluent and noneffluent fixed points and could be omitted in the definition of inessential fixed points. But it is, in the light of some work on trees by L. E. Ward, Jr. [12], quite possible that at least $\$ 5$ will not hold for nonmetric trees. An attempt to prove the results of $\$ 3$ and $\$ 4$ without the help of a metric seems to have a better chance of success.

I would like to thank the referee for his helpful suggestions.

2. Different types of fixed points on dendrites. Let us first recollect the definition of a dendrite, and those of its properties needed in this paper.

A dendrite $D$ is a metric continuum in which every pair of distinct points is separated by a third one. $D$ has a partial order structure which was developed by L. E. Ward, Jr. [9], [10]. It is obtained by selecting an arbitrary point $a \in D$ as root, and defining $x \leqq y$ if $x=a, x$ separates $a$ 
and $y$, or $x=y$. Then $a \leqq x$ for all $x \in D$. The sets

$$
L(x)=\{y \in D \mid y \leqq x\}
$$

and

$$
M(x)=\{y \in D \mid x \leqq y\}
$$

are closed in $D$, the set $M(x) \backslash\{x\}$ is open, and $L(x)$ is a chain (i.e., is linearly ordered). A point $m$ is called a maximum (minimum) of a subset $A$ of $D$ if $m \nless x(x \nless m)$ for each $x \in A$. It was proved by Ward ([9], Theorem $1 ;$ [10], Theorem 1 ) that every nonempty closed subset of $D$ has a maximum and a minimum. As $L(x)$ is a chain, the maximum and minimum of a closed nonempty subset of $L(x)$ is unique. So is the minimum of a subdendrite ([3], Lemma 2).

It follows from [13], pp. 88-89, that $D$ is locally connected and that every connected subset is arc-connected. The arc $[x, y]$ between any two points $x, y \in D$ is unique, and consists of all points which separate $x$ and $y$. Every closed and connected subset of $D$ is a subdendrite ([13], p. 89), and hence $M(x)$ and $(D \backslash M(x)) \cup\{x\}$ are subdendrites. A dendrite has, at each of its points, a neighbourhood basis $\mathcal{N}$ so that every $N \in \mathcal{N}$ is a subdendrite with a finite boundary. (As $D$ is regular ([4], p. 301), there exists for every neighbourhood $U$ of a point $x \in D$ a neighbourhood $V$ of $x$ which has a finite boundary, and we can also ask that its closure $\mathrm{Cl} V$ is contained in $U$. A neighbourhood $N \in \mathcal{N}$ with $N \subset U$ can then be obtained as the closure of the arc-component of $V$ which contains $x$.) We call the elements $N \in \mathcal{N}$ the basic neighbourhoods of $D$ at $x$.

The order $o(x)$ of a point $x$ of $D$ is defined in [13], p. 48. The point $x$ is called an endpoint if its order is one, and a branchpoint if its order is greater than two. If either the order of $x$ or the number of components of $D \backslash\{x\}$ is finite, then these two numbers are equal ([13], p. 88).

It is the main purpose of this paper to construct mappings with given effluent and noneffluent fixed points. We now define these, as well as inessential fixed points. All definitions apply only to a fixed point $c$ of $f: D \rightarrow D$ which is isolated, i.e., which has a neighbourhood $U$ such that $\mathrm{Cl} U \cap \operatorname{Fix} f=\{c\}$, where Fix $f$ denotes the fixed point set of $f$. We give $D$ a convex metric $d$ [1], [6], and denote the boundary of a subset by Bd.

Definition 2.1. Let $D$ be a dendrite and $f: D \rightarrow D$ be a map with fixed point set Fix $f$. An isolated fixed point $c \in$ Fix $f$ is called inessential if for every neighbourhood $U$ of $c$ with $\mathrm{Cl} U \cap$ Fix $f=\{c\}$ and every $\epsilon>0$ there exists a map $g: D \rightarrow D$ such that 
(i) $d(f(x), g(x))<\epsilon$ for all $x \in D$,

(ii) $g(x)=f(x)$ for all $x \in D \backslash U$,

(iii) $g$ is fixed point free on $\mathrm{CIU}$.

Otherwise an isolated fixed point is called essential.

Definition 2.2. Let $D$ be a dendrite and $f: D \rightarrow D$ be a map with fixed point set Fix $f$. A fixed point $c \in$ Fix $f$ is called effluent if every neighbourhood of $c$ contains a basic neighbourhood $N(c)$ such that $N(c) \cap \operatorname{Fix} f=\{c\}$ and such that $x$ separates $c$ and $f(x)$ whenever $x \in \operatorname{Bd} N(c)$. A fixed point $c \in$ Fix $f$ is called noneffluent if every neighbourhood of $c$ contains a basic neighbourhood $N(c)$ such that $N(c) \cap$ Fix $f=\{c\}$ and such that $x$ does not separate $c$ and $f(x)$ whenever $x \in \mathrm{Bd} N(c)$.

We see that a source of a vector field corresponds to an effluent fixed point, and that sinks and circulations correspond to noneffluent fixed points. - It should be noted that noneffluence is a stronger condition than the negation of effluence and that the term "strictly noneffluent" would have been more precise. We have avoided this pedanticism as confusion seems unlikely.

3. Some properties of noneffuent fixed points. We will show in this paragraph that every noneffluent fixed point is essential (Theorem 3.2), prove that there always exists at least one noneffluent fixed point (Theorem 3.5), and finally state a criterion for the existence of exactly one noneffluent fixed point (Theorem 3.6). A frequent tool used in the proofs here and further on is a retraction of the dendrite $D$ onto a given subdendrite $D_{0}$.

LEMMA 3.1. Let $D_{0}$ be a subdendrite of the dendrite $D$ which contains the root of $D$. Then the function $r: D \rightarrow D_{0}$ defined by

$$
r(x)=\max \left(L(x) \cap D_{0}\right) \quad \text { for all } x \in D
$$

is a retraction, and $r(x) \in \mathrm{Bd} D_{0}$ if $x \notin D_{0}$.

Proof. The function $r$ was used by L. Lum in [5], Theorem 2.1, and it is shown there that it is a retraction. From the definition of $r(x)$ as a maximum it is immediate that $r(x) \in \mathrm{Bd} D_{0}$ if $x \notin D_{0}$.

With the help of this lemma, the proof of the essential character of every noneffluent fixed point is simple.

THEOREM 3.2. Every noneffluent fixed point of a dendrite is essential. 
Proof. Let $a$ be a noneffluent fixed point of the selfmap $f: D \rightarrow D$. We choose it as the root of $D$. As $a$ is noneffluent, we can find a basic neighbourhood $N(a)$ such that $N(a) \cap$ Fix $f=\{a\}$ and $x \notin(a, f(x))$ for $x \in \operatorname{Bd} N(a)$, where $(a, f(x))=[a, f(x)] \backslash\{a, f(x)\}$. Let $r: D \rightarrow N$ be the retraction of Lemma 3.1.

Assume now that $a$ is inessential, so that there exists a map $g: D \rightarrow D$ with $g(x)=f(x)$ for all $x \in D \backslash N(a)$ which is fixed point free on $N(a)$. Define $f^{\prime}: N(a) \rightarrow N(a)$ by $f^{\prime}=r \circ g \mid N(a)$. If $x \notin \operatorname{Bd} N(a)$ and $g(x) \in N(a)$ then $f^{\prime}(x)=g(x) \neq x$, if $x \notin \mathrm{Bd} N(a)$ and $g(x) \notin N(a)$ then $f^{\prime}(x) \in \operatorname{Bd} N(a)$, so in either case $f^{\prime}(x) \neq x$. If $x \in \operatorname{Bd} N(a)$, then $f^{\prime}(x)=r \circ f(x)$ and $x \notin(a, f(x))$. Therefore $r \circ f(x) \leqq f(x)$ implies $f^{\prime}(x) \neq x$. Hence $f^{\prime}$ is a fixed point free selfmap of the dendrite $N(a)$, which contradicts the fact that $N(a)$ has the fixed point property. So $a$ must be essential.

We now want to establish the existence of a noneffluent fixed point. This will involve us in a more complicated proof, and we prepare for it with two lemmas.

Lemma 3.3. For every $f: D \rightarrow D$ the set $E=\{x \in D \mid x \leqq f(x)\}$ is closed in $D$.

Proof. This follows e.g. from [11], Lemma 7.

LEMMA 3.4. If $x^{\prime}, x^{\prime \prime} \in D$ are two points such that $x^{\prime \prime} \in\left(x^{\prime}, f\left(x^{\prime \prime}\right)\right)$ and $x^{\prime} \in\left(x^{\prime \prime}, f\left(x^{\prime}\right)\right)$, then $f$ has a fixed point on $\left(x^{\prime}, x^{\prime \prime}\right)$.

Proof. Take $f\left(x^{\prime}\right)$ as root. Then by assumption $f\left(x^{\prime}\right)<x^{\prime}<x^{\prime \prime}<$ $f\left(x^{\prime \prime}\right)$. The set $E_{0}=E \cap\left[x^{\prime}, x^{\prime \prime}\right]$ is closed by Lemma 3.3, is nonempty as $x^{\prime \prime} \in E_{0}$, and is $\neq\left[x^{\prime}, x^{\prime \prime}\right]$ as $x^{\prime} \notin E_{0}$. Let $x_{0}=\min E_{0}$. As $E_{0}$ is closed, $x_{0} \in E_{0}$, so that $x_{0} \leqq f\left(x_{0}\right)$. Assume now that $x_{0}<f\left(x_{0}\right)$. The partial order of $D$ is order dense [10], therefore we can choose $t$ with $x_{0}<t<f\left(x_{0}\right)$, and, as $f$ is continuous, an open set $U\left(x_{0}\right)$ containing $x_{0}$ and such that $f\left(U\left(x_{0}\right)\right) \subset M(t) \backslash\{t\}$. But then $x \in E_{0}$ for all $x \in L\left(x_{0}\right) \cap$ $U\left(x_{0}\right)$, contradicting the minimality of $x_{0}$. So we must have $x_{0}=f\left(x_{0}\right)$, and $x_{0}$ is the desired fixed point.

THEOREM 3.5. If a selfmap of a dendrite has a finite fixed point set, then it has at least one noneffluent fixed point.

Proof. Let $f=f_{1}: D \rightarrow D$ be a selfmap of a dendrite $D=D_{1}$ with a finite fixed point set Fix $f$. As Fix $f \neq \varnothing$, we can choose a point $a_{1} \in$ Fix $f$ as root. If $a_{1}$ is not as desired, then there exists a basic neighbourhood 
$N_{1}\left(a_{1}\right)$ with $N_{1}\left(a_{1}\right) \cap$ Fix $f=\left\{a_{1}\right\}$, and such that $a_{1}<x_{1}<f_{1}\left(x_{1}\right)$ for at least one $x_{1} \in \operatorname{Bd} N_{1}\left(a_{1}\right)$. Now consider the set $E_{1}=\left\{x \in D_{1} \mid x \leqq f_{1}(x)\right\}$. As $x_{1} \in E_{1}$, we have $E_{1} \neq\left\{a_{1}\right\}$. An argument similar to the one used in the proof of Lemma 3.4 shows that no $x$ with $x<f_{1}(x)$ can be a maximal element of $E_{1}$. But $E_{1}$ is closed by Lemma 3.3, and hence must contain at least one maximal element, $a_{2}$ say. Then $a_{2} \in E_{1} \cap$ Fix $f$, and as $x_{1} \in E_{1}$, we have $a_{2} \neq a_{1}$.

Assume now that $a_{2}$ is again not as desired. We shall show that in this case there must exist a third fixed point $a_{3}$. For this purpose, select a basic neighbourhood $N_{2}\left(a_{2}\right)$ with $N_{2}\left(a_{2}\right) \cap$ Fix $f=\left\{a_{2}\right\}$, such that $x_{2} \in\left(a_{2}, f_{1}\left(x_{2}\right)\right)$ for at least one $x_{2} \in \mathrm{Bd} N_{2}\left(a_{2}\right)$. If $x_{2} \in M\left(a_{2}\right) \backslash\left\{a_{2}\right\}$, then $a_{2}<x_{2}<f_{1}\left(x_{2}\right)$ implies $x_{2} \in E_{1}$ which contradicts the maximality of $a_{2}$. Hence $x_{2} \in \operatorname{Bd} N_{2}\left(a_{2}\right) \cap\left(D \backslash M\left(a_{2}\right)\right)$. Let $y_{2}=\max \left(L\left(a_{2}\right) \cap L\left(x_{2}\right)\right)$, so that $a_{1}<y_{2}<a_{2}$, and denote by $K\left(a_{2}\right)$ the component of $D_{1} \mid\left\{y_{2}\right\}$ which contains $a_{2}$. As $y_{2} \in\left[a_{2}, x_{2}\right]$, we have $y_{2} \in N_{2}\left(a_{2}\right)$ and therefore $f_{1}\left(y_{2}\right) \neq y_{2}$. We also have $f_{1}\left(y_{2}\right) \notin K\left(a_{2}\right)$, for otherwise the assumptions of Lemma 3.4 would be satisfied for $x_{2}, y_{2}$ (note that $f_{1}\left(y_{2}\right) \in K\left(a_{2}\right)$ implies $\left.y_{2} \neq x_{2}\right)$ and $f_{1}$ would have a fixed point on $\left(x_{2}, y_{2}\right) \subset N_{2}\left(a_{2}\right)$, in contradiction to $N_{2}\left(a_{2}\right) \cap$ Fix $f=\left\{a_{2}\right\}$. Now let $D_{2}=D_{1} \backslash K\left(a_{2}\right)$, and define $f_{2}: D_{2} \rightarrow D_{2}$ by $f_{2}=r_{2} \circ f_{1} \mid D_{2}$, where $r_{2}: D_{1} \rightarrow D_{2}$ is the retraction from Lemma 3.1. As $a_{1}$ is not a noneffluent fixed point of $f_{1}$, it is not a noneffluent fixed point of $f_{2}$. We can therefore repeat the argument from the beginning of the proof for $f_{2}$ and $E_{2}=\left\{x \in D_{2} \mid x \leqq f_{2}(x)\right\}$ instead of $f_{1}$ and $E_{1}$, and find a maximal element $a_{3}$ of $E_{2}$. Again $E_{2} \neq\left\{a_{1}\right\}$ : if $y_{2} \notin E_{2}$, then $y_{2} \in D_{2}$ implies $y_{2} \nless f_{1}\left(y_{2}\right)$. If also $x_{1} \notin E_{2}$, then $x_{1}<f_{1}\left(x_{1}\right)$ implies $x_{1} \notin D_{2}$ and hence $y_{2}<x_{1}$. But then Lemma 3.4 yields a fixed point of $f_{1}$ on $\left(y_{2}, x_{1}\right)$ which must be different from $a_{1}$, and this cannot happen as now $\left(y_{2}, x_{1}\right) \subset N_{1}\left(a_{1}\right)$ and $N_{1}\left(a_{1}\right) \cap$ Fix $f=$ $\left\{a_{1}\right\}$. Therefore $E_{2}$ must contain at least one of $x_{1}$ and $y_{2}$, and we have $a_{3} \in$ Fix $f, a_{3} \neq a_{1}$, and also $a_{3} \neq a_{2}$ and $a_{2} \notin E_{2}$.

If $a_{3}$ is again not as desired, we repeat the last step of the proof, and obtain a fixed point $a_{4} \in D_{3}=D_{2} \backslash K\left(a_{3}\right)$, where $K\left(a_{3}\right)$ is defined in $D_{2}$ analogously to $K\left(a_{2}\right)$ in $D_{1}$, and such that $a_{4} \in \operatorname{Fix} f \backslash\left\{a_{1}, a_{2}, a_{3}\right\}$. This process, if continued, must eventually lead to a noneffluent fixed point as the fixed point set Fix $f$ is finite, and therefore Theorem 3.5 must be true.

We finally state a result which shows that usually more than one noneffluent fixed point can be found.

THEOREM 3.6. Let $f$ be a selfmap of a dendrite with a finite fixed point set. Then $f$ has exactly one noneffluent fixed point if and only if all but one of its fixed points are inessential.

The proof of Theorem 3.6 is lengthy and uses similar methods to 
those employed in the proofs of Theorems 3.2 and 3.5. It is omitted as the theorem is not used in the rest of this paper.

\section{Some properties of effluent fixed points. We now} turn our attention to effluent fixed points. An effluent fixed point need not be essential, but we can show that it is inessential if and only if it is an endpoint (Theorem 4.1). There is no counterpart to Theorem 3.5, as effluent fixed points need not exist. But if they do, then the number of noneffluent fixed points increases. We establish in Theorems 4.2 and 4.4 two lower bounds for the number of noneffluent fixed points for maps which have effluent fixed points.

THEOREM 4.1. An effluent fixed point of a selfmap of a dendrite is inessential if and only if it is an endpoint.

Proof. (i) Let $b$ be an effluent and inessential fixed point of the selfmap $f$ of the dendrite $D$, and Fix $f$ be its (not necessarily finite) fixed point set. We use $b$ as root. Assume by way of contradiction that $b$ is not an endpoint, so that $D \backslash\{b\}$ has at least two components $K_{1}$ and $K_{2}$. As $b$ is effluent we can find a basic neighbourhood $N(b)$ with the following properties: $N(b) \nsupseteq K_{t}$ for $i=1,2, N(b) \cap \operatorname{Fix} f=\{b\}$, and $x<$ $f(x)$ for all $x \in \operatorname{Bd} N(b)$. If $y_{t} \in K_{i} \backslash N(b)$, then the arc $\left[b, y_{i}\right]$ is contained in the arc-connected set $K_{t} \cup\{b\}$. From $b \in N(b)$ and $y_{1} \notin N(b)$ it follows that there exists $x_{1} \in\left(b, y_{1}\right) \cap \operatorname{Bd} N(b)$ for $i=1,2$. As $b$ is inessential, we can find a selfmap $g$ of $D$ such that $g(x)=f(x)$ for all $x \in D \backslash N(b)$ and such that $g$ is fixed point free on $N(b)$. But then $g\left(x_{i}\right)=f\left(x_{i}\right)$ and therefore $x_{i}<g\left(x_{i}\right)$ for $i=1,2$, so that Lemma 3.4 asserts that $g$ has a fixed point on $\left(x_{1}, x_{2}\right) \subset N(b)$. Hence we arrive at a contradiction.

(ii) Now consider an effluent fixed point $b \in D$ which is an endpoint, and select it as root. Choose any $\epsilon>0$, and any neighbourhood $U(b)$ with $\operatorname{Cl} U(b) \cap$ Fix $f=\{b\}$. Take $\delta>0$ such that $\operatorname{diam} P<\delta$ implies diam $f(P)<\epsilon$ for all $P \subset D$. As $b$ is effluent there exists a basic neighbourhood $N(b) \subset U(b)$ such that $x<f(x)$ for all $x \in \mathrm{Bd} N(B)$; we can also require that $\operatorname{diam} N(b)<\delta$ and $N(b) \neq D$. As $b$ is an endpoint, we can choose an open neighbourhood $V(b)$ with $\mathrm{Cl} V(b) \subset N(b)$ and such that $\mathrm{Bd} V(b)$ consists of exactly one point $x_{1}$. Let $K(b)$ be the component of $V(b)$ which contains $b$. Then $K(b)$ is open in $V(b)$, $\operatorname{Bd} K(b)=\left\{x_{1}\right\}$, and it is easy to see that $K(b) \cup M\left(x_{1}\right)=D$. As $K(b) \cup M\left(x_{1}\right)=D$ and $K(b) \subset N(b) \neq D$, there exists a point $x^{\prime} \in$ $M\left(x_{1}\right) \backslash N(b)$, and therefore a point $x_{2} \in\left[x_{1}, x^{\prime}\right] \cap \mathrm{Bd} N(b)$. Then $x_{2}<$ $f\left(x_{2}\right)$, and $f$ is fixed point free on $\left(x_{1}, x_{2}\right) \subset N(b)$, so that Lemma 3.4 implies $x_{1}<f\left(x_{1}\right)$. We see that $f\left(x_{1}\right) \notin K(b)$, for otherwise $x_{1} \in$ 
$\left[b, f\left(x_{1}\right)\right] \subset K(b)$. But $x_{1}$ is the boundary of the open set $K(b)$, so $x_{1} \notin K(b)$.

We define $g: D \rightarrow D$ by

$$
f(x)= \begin{cases}f\left(x_{1}\right) & \text { if } x \in \mathrm{Cl} K(b) \\ f(x) & \text { if } x \in D \backslash K(b)\end{cases}
$$

Then $g$ is continuous, $d(f(x), g(x))<\epsilon, g(x)=f(x)$ for $x \in D \backslash U(b)$ and $g$ is fixed point free on $\mathrm{Cl} U(b)$. Hence $b$ is inessential.

It was not necessary in Theorem 4.1 to assume that $f$ has a finite fixed point set, but in the following theorem the assumption is needed.

THEOREM 4.2. If $b$ is an effluent fixed point of a selfmap of $a$ dendrite $D$ with a finite fixed point set, then every component of $D \backslash\{b\}$ contains at least one noneffluent fixed point.

Proof. Take the effluent fixed point $b$ of the map $f: D \rightarrow D$ with fixed point set Fix $f$ as root, and let $K$ be a component of $D \backslash\{b\}$. As $D_{0}=K \cup\{b\}$ is a subdendrite, a retraction $r: D \rightarrow D_{0}$ can be defined, as in Lemma 3.1, by $r(x)=x$ if $x \in D_{0}$ and $r(x)=b$ if $x \notin D_{0}$. It follows from Theorem 3.5 that the map $g: D_{0} \rightarrow D_{0}$ given by $g(x)=r \circ f(x)$ for all $x \in D_{0}$ has a noneffluent fixed point $a$. If $a \neq b$, then the theorem is proved, as a noneffluent fixed point of $g$ is clearly a noneffluent fixed point of $f$ if it is contained in the open subset $K$ of $D$. It remains to rule out the possibility that $a=b$.

If $a=b$ and $b$ is effluent for $f$, then we can select a neighbourhood $U(b)$ in $D$ with $U(b) \cap$ Fix $f=\{b\}$ which contains a basic neighbourhood $N(b)$ with $x<f(x)$ for $x \in \operatorname{Bd} N(b)$, and such that $K \not \subset N(b)$. Then $K \cap \operatorname{Bd} N(b) \neq \varnothing$, so that there exists an $x_{1} \in K \cap \operatorname{Bd} N(b)$ with $x_{1}<$ $f\left(x_{1}\right)$. Let $U^{\prime}(b)$ be the neighbourhood of $b$ in $D_{0}$ which consists of all points $x$ with $d(b, x)<\frac{1}{2} d\left(b, x_{1}\right)$. As $a$ is noneffluent for $g, U^{\prime}(b)$ contains a basic neighbourhood $N^{\prime}(b)$ of $b$ in $D_{0}$ with $x \nless g(x)$ for $x \in \mathrm{Bd}_{D_{0}} N^{\prime}(b)$, where $\mathrm{Bd}_{D_{0}}$ denotes the boundary in $D_{0}$. The fact that $x_{1} \notin N^{\prime}(b)$ allows us to choose $x_{2} \in \mathrm{Bd}_{D_{0}} N^{\prime}(b) \cap\left(b, x_{1}\right)$, and $x_{2} \nless g\left(x_{2}\right)$ implies $x_{2} \nless f\left(x_{2}\right)$. Therefore Lemma 3.4 yields a fixed point of $f$ on $\left(x_{1}, x_{2}\right) \subset N(b) \subset U(b)$, in contradiction to $U(b) \cap$ Fix $f=\{b\}$. So $a=b$ is impossible.

COROLlaRY 4.3. If $b$ is an effluent fixed point of a selfmap of $a$ dendrite with a finite fixed point set, then the order of $b$ is finite.

Proof. If $b$ is not of finite order, then $D \backslash\{b\}$ has infinitely many components. But Theorem 4.2 asserts the existence of a noneffluent 
fixed point in each of them, which cannot happen if the fixed point set of $f$ is finite.

Note that the counterpart of Corollary 4.3 for noneffluent fixed points is not true. If the order of the point $a \in D$ is not finite, then the map $f: D \rightarrow D$ given by $f(x)=a$ for all $x \in D$ has $a$ as its only fixed point, and $a$ is noneffluent. Less trivial counterexamples can also be easily constructed.

Theorem 4.2 provides information about the location as well as the number of noneffluent fixed points, and the lower bound for the number of noneffluent fixed points obtained in it is the best possible one if only one effluent fixed point is present. But we shall now sharpen this bound considerably for the case where more than one effluent fixed point exists. We denote by \# $A$ the number of elements of the finite set $A$.

THEOREM 4.4. Let a selfmap of a dendrite have a finite fixed point set, and let $A$ be the set of its noneffluent fixed points and $B$ be the set of its effluent fixed points. Then

$$
\# A \geqq 1+\sum_{b \in B}(o(b)-1) .
$$

Proof. We know from Theorem 3.5 that $A \neq \varnothing$, so we can select a noneffluent fixed point $a_{0}$ as root. Let now $b_{t} \in B$ be an effluent fixed point of the selfmap $f$ of order $o\left(b_{l}\right)$. It follows from Corollary 4.3 that $o\left(b_{1}\right)$ is finite, so that $D \backslash\left\{b_{l}\right\}$ has $o\left(b_{l}\right)$ components. Let $K_{t k}(k=$ $\left.1,2, \cdots, o\left(b_{t}\right)-1\right)$ be the components of $D \backslash\left\{b_{l}\right\}$ which do not contain $a_{0}$.

For each $K_{i k}$ the set

$$
D_{i k}=\left[K_{i k} \cup\left\{b_{l}\right\}\right] \backslash\left[\cup\left(M\left(b_{l}\right) \backslash\left\{b_{\jmath}\right\} \mid b_{\jmath} \in K_{i k}\right)\right]
$$

(where the set in the second square bracket can be empty) is a subdendrite of $D$, and the function $r_{t k}: D \rightarrow D_{\imath k}$ given by

$$
r_{t k}(x)=\left\{\begin{array}{lll}
x & \text { if } & x \in D_{i k}, \\
b_{t} & \text { if } & x \in D \backslash K_{t k}, \\
b_{l} & \text { if } & x \in M\left(b_{l}\right) \text { and } b_{l} \in K_{t k}
\end{array}\right.
$$

is a retraction. Define

$$
g_{i k}: D_{\imath k} \rightarrow D_{\imath k} \quad \text { by } \quad g_{i k}(x)=r_{i k} \circ f(x)
$$

for all $x \in D_{i k}$. It follows from Theorem 3.5 that $g_{i k}$ has at least one 
noneffluent fixed point, say $a_{k k}$, and an argument similar to the one used in the proof of Theorem 4.2 shows that $a_{t k} \neq b_{t}$ and $a_{t k} \neq b_{\jmath}$ for all $b_{t} \in K_{t k}$. Therefore $a_{t k}$ is also a noneffluent fixed point of $f$. In this way, we associate with each effluent fixed point $b_{1}$ a set of $o\left(b_{1}\right)-1$ noneffluent fixed points $a_{t k}$, which are different from the noneffluent fixed point $a_{0}$.

It remains to show that all $a_{t k}$ are different. As $b_{t}<a_{i k}$, the fact that $a_{t k}=a_{\jmath t}$ would imply $b_{t}<b_{\jmath}$ or $b_{l}<b_{t}$, say $b_{t}<b_{\jmath}$. But then $b_{t}<b_{l}<a_{t k}$, which is not possible according to the construction of the $a_{t k}$ in $D_{t k}$. So $a_{0}$ and the points $a_{t k}$ form a set of $1+\Sigma_{b \in B}(o(b)-1)$ noneffluent fixed points of $f$.

REMARK. In general, $f$ has in fact more noneffluent fixed points than the minimum number required by Theorem 4.4. As an example, consider the cross in the $x y$-plane given by

$$
D=\left\{(x, y) \in R^{2}|| x \mid \leqq 1 \text { and } y=0, \text { or } x=0 \text { and }|y| \leqq 1\right\}
$$

and define $f: D \rightarrow D$ by

$$
\begin{array}{ll}
f(x, 0)=\left(\frac{3}{2} x-\frac{1}{2}|x| x, 0\right) & \text { for all }(x, 0) \in D, \\
f(0, y)=\left(0, \frac{1}{2} y+\frac{1}{2}|y| y\right) & \text { for all }(0, y) \in D
\end{array}
$$

(i.e. $f$ moves the points on the $x$-axis away from the origin, and the points on the $y$-axis towards the origin).

The selfmap $f$ has five fixed points at $(0,0),( \pm 1,0)$, and $(0, \pm 1)$. The points $( \pm 1,0)$ are noneffluent and the points $(0, \pm 1)$ are effluent and all are of order one. The point $(0,0)$ is neither effluent nor noneffluent. Hence

$$
\# A=2>1+\sum_{b \in B}(o(b)-1)=1
$$

But we will show in the next paragraph, in Theorem 5.2, that $f$ has precisely $1+\Sigma_{b \in B}(o(b)-1)$ noneffluent fixed points if $f$ has a finite fixed point set and all fixed points of $f$ are either effluent or noneffluent.

\section{Mappings of dendrites with prescribed effuent and} noneffiuent fixed points. The last paragraph of this paper contains its main result, the construction of a selfmap of a dendrite with a prescribed set of effluent and noneffluent fixed points. Necessary and sufficient conditions for the existence of such maps will be given in Theorem 5.2. They are consequences of Corollary 4.3 and Theorem 4.4, 
supplemented by one further condition which is motivated by Lemma 5.1. The proof of Theorem 5.2 depends heavily on the fact that $D$ has a convex metric.

LemMA 5.1. Let $A \neq \varnothing$ and $B$ be two finite subsets of $a$ dendrite, and let the order $o(b)$ be finite for each $b \in B$. If

$$
\left(a^{\prime}, a^{\prime \prime}\right) \cap B \neq \varnothing \quad \text { for all distinct } a^{\prime}, a^{\prime \prime} \in A,
$$

then

$$
\# A \leqq 1+\sum_{b \in B}(o(b)-1)
$$

Proof. Select an element $a_{1} \in A$ as root of the dendrite $D$, and index the elements of $A$ so that $L\left(a_{t}\right)$ does not contain more elements of $B$ than $L\left(a_{1}\right)$ whenever $i<j$. This obviously implies $a_{j} \nless a_{v}$. Let, for all $k=2,3, \cdots, \# A$, the finite subdendrite $D_{k}$ of $D$ be given by $D_{k}=\cup\left(\left[a_{1}, a_{l}\right] \mid i=2,3, \cdots, k\right)$, and denote by $o_{k}\left(b_{j}\right)$ the order of $b_{j}$ in $D_{k}$. We shall proceed by induction on $k$, and associate with each $a_{k}$ an element $b_{k} \in B \cap D_{k}$ of order $o_{k}\left(b_{k}\right) \geqq 2$, so that either $b_{k} \neq b_{1}$ for all $j<k$, or $o_{k}\left(b_{l}\right)=o_{k-1}\left(b_{j}\right)+1$ for some $j<k$. If the points $a_{1}, a_{2}, \cdots, a_{k-1}$ and $b_{2}, b_{3}, \cdots, b_{k-1}$ satisfy

$$
k-1 \leqq 1+\sum_{j \leqq k-1}\left(o_{k-1}\left(b_{l}\right)-1\right),
$$

then the points $a_{1}, a_{2}, \cdots, a_{k}$ and $b_{2}, b_{3}, \cdots, b_{k}$ satisfy

$$
k \leqq 1+\sum_{\jmath \leqq k}\left(o_{k}\left(b_{l}\right)-1\right)
$$

As $o_{k}(b) \leqq o(b)$ for all $b \in B$ and as $\left\{b_{l} \mid 2 \leqq j \leqq \# A\right\} \subseteq B$, this gives the desired result (i).

If $k=2$, then $\left(a_{1}, a_{2}\right) \cap B \neq \varnothing$ allows us to choose $b_{2} \in\left(a_{1}, a_{2}\right)$, and as $o_{2}\left(b_{2}\right)=2$, we see that (ii) holds for $k=2$.

Now assume that (ii) holds for the $k-1$ points $a_{1}, a_{2}, \cdots, a_{k-1}$ in $D_{k-1}$. Let $m_{k}=\max \left(L\left(a_{k}\right) \cap D_{k-1}\right) \in D_{k-1}$. If $m_{k} \in B \cap D_{k-1}$, then we choose $b_{k}=m_{k}$. In this case we either have $b_{k}=b_{j}$ for some $j<k$, and hence $o_{k}\left(b_{k}\right)=o_{k-1}\left(b_{k}\right)+1$, or $b_{k} \neq b_{j}$ for all $j<k$. If $m_{k} \in A \cap D_{k-1}$, then $\left(m_{k}, a_{k}\right) \cap B \neq \varnothing$, and we can select $b_{k} \in\left(m_{k}, a_{k}\right)$. If finally $m_{k} \notin(A \cup B) \cap D_{k-1}$, then there exists an $a_{l} \in D_{k-1}$ with $m_{k}<a_{l}$. It cannot happen that $\left(m_{k}, a_{k}\right) \cap B \neq \varnothing$, for $\left(a_{l}, a_{k}\right)=\left(a_{l}, m_{k}\right] \cup\left[m_{k}, a_{k}\right)$ must 
intersect $B$, and $\left(m_{k}, a_{t}\right) \cap B \neq \varnothing$ with $\left(m_{k}, a_{k}\right) \cap B \neq \varnothing$ and $i<k$ contradicts the way in which $A$ was indexed. So we can again choose $b_{k} \in\left(m_{k}, a_{k}\right)$. We see that in each of these possible cases (ii) is satisfied for $a_{1}, a_{2}, \cdots, a_{k}$.

We now proceed to establish the criteria for maps with prescribed sets of effluent and noneffluent fixed points.

Theorem 5.2. Let $A$ and $B$ be two finite subsets of $a$ dendrite. Then there exists a selfmap with fixed point set $A \cup B$ for which all points in $A$ are noneffluent and all points in $B$ are effluent, if and only if the following three conditions hold:

(i) The order $o(b)$ is finite for each $b \in B$,

(ii) $\left(a^{\prime}, a^{\prime \prime}\right) \cap B \neq \varnothing$ for all distinct $a^{\prime}, a^{\prime \prime} \in A$,

(iii) $\# A \geqq 1+\Sigma_{b \in B}(o(b)-1)$.

Proof. A. Necessity. Condition (i) follows from Corollary 4.3 and (iii) follows from Theorem 4.4, so we only have to deal with (ii). Assume therefore that a selfmap $f: D \rightarrow D$ of a dendrite $D$ exists with the prescribed fixed point set, and that there are two distinct points $a^{\prime}, a^{\prime \prime} \in A$ for which $\left(a^{\prime}, a^{\prime \prime}\right) \cap B=\varnothing$. We can choose a root of $D$ such that $a^{\prime}<a^{\prime \prime}$, and can clearly also assume that $\left(a^{\prime}, a^{\prime \prime}\right) \cap A=\varnothing$. As $a^{\prime}$ and $a^{\prime \prime}$ are noneffluent we can with the help of suitable neighbourhoods find points $x^{\prime}, x^{\prime \prime} \in\left(a^{\prime}, a^{\prime \prime}\right)$ with $a^{\prime}<x^{\prime}<x^{\prime \prime}<a^{\prime \prime}$ and so that $x^{\prime} \notin\left(a^{\prime}, f\left(x^{\prime}\right)\right)$ and $x^{\prime \prime} \notin\left(a^{\prime \prime}, f\left(x^{\prime \prime}\right)\right)$. But then $x^{\prime \prime} \in\left(x^{\prime}, f\left(x^{\prime \prime}\right)\right)$ and $x^{\prime} \in$ $\left(x^{\prime \prime}, f\left(x^{\prime}\right)\right)$, so Lemma 3.4 asserts that $f$ has a fixed point on $\left(x^{\prime}, x^{\prime \prime}\right)$. This cannot happen if $A \cup B$ is the fixed point set of $f$, and (ii) must hold.

B. Sufficiency. Assume now that Fix $f=A \cup B$, is a finite subset of $D$ which satisfies conditions (i), (ii), and (iii). As \# $A \geqq 1$, we can index the points in $A$ as in the proof of Lemma 5.1, and see from Lemma 5.1 (i) and Theorem 5.2 (iii) that the points $b_{k}$ obtained in this proof are all the points of $B$ apart from possibly some points of order one, i.e., we have $B=B^{\prime} \cup B^{\prime \prime}$, where $B^{\prime}=\left\{b_{1} \mid 2 \leqq j \leqq \# A\right\}$ with $o\left(b_{1}\right) \geqq 2$ and not necessarily all $b$ distinct, and where $o(b)=1$ for all $b \in B^{\prime \prime}$. We also have, in the notation of the proof of Lemma 5.1, o(b)=o $o_{* A}(b)$ for all $b \in B^{\prime}$. Let $D_{k}$, for $2 \leqq k \leqq \# A$, be the subdendrite from the proof of Lemma 5.1, and $D_{1}=\left\{a_{1}\right\}$. We first define maps $f_{k}: D_{k} \cup A \cup$ $B^{\prime} \rightarrow D_{k} \cup A \cup B^{\prime}$ with fixed point set $A \cup B^{\prime}$ inductively for $k=$ $1,2, \cdots, \# A$.

If $k=1$, then $D_{1} \cup A \cup B^{\prime}=A \cup B^{\prime}$, therefore we put $f_{1}(x)=x$ for all $x \in D_{1} \cup A \cup B^{\prime}$. Now assume that $f_{k-1}: D_{k-1} \rightarrow D_{k-1}$ has been defined. Let again $m_{k}=\max \left(L\left(a_{k}\right) \cap D_{k-1}\right)$, so that $D_{k}=$ $D_{k-1} \cup\left[m_{k}, a_{k}\right]$, where $\left[m_{k}, a_{k}\right]=\left[m_{k}, b_{k}\right] \cup\left[b_{k}, a_{k}\right]$, with $m_{k}=b_{k}$ or 
$m_{k} \neq b_{k}$, but always $b_{k} \neq a_{k}$. In the definition of $f_{k}$ we shall use parameters $\lambda$ and $\mu$, with $0 \leqq \lambda \leqq 1$ and $\mu=\lambda+\frac{1}{2} \lambda(1-\lambda)$. Note that then $0 \leqq \mu \leqq 1$, and $\mu \geqq \lambda$ with $\mu=\lambda$ if and only if $\lambda=0$ or $\lambda=1$. We put

$$
f_{k}(x)= \begin{cases}f_{k-1}(x) & \text { if } x \in D_{k-1} \cup A \cup B^{\prime} \\ \mu a_{k}+(1-\mu) b_{k} & \text { if } x=\lambda a_{k}+(1-\lambda) b_{k}, \\ \mu f_{k-1}\left(m_{k}\right)+(1-\mu) b_{k} & \text { if } x=\lambda m_{k}+(1-\lambda) b_{k}\end{cases}
$$

We also write $D^{\prime}=D_{\# A}=D_{\# A} \cup A \cup B^{\prime}$ and $f^{\prime}: D^{\prime} \rightarrow D^{\prime}$ for the case $k=\# A$. It is easy to check that $f^{\prime}$ is continuous, that its fixed point set is Fix $f=A \cup B^{\prime}$, and that the map $f^{\prime}: D^{\prime} \rightarrow D^{\prime}$ has the points of $A$ as noneffluent fixed points and the points of $B^{\prime}$ as effluent fixed points.

If $B^{\prime \prime}=\varnothing$, let $D^{\prime \prime}=D^{\prime}$ and $f^{\prime \prime}=f^{\prime}$. If $B^{\prime \prime} \neq \varnothing$, i.e., if a map with inessential effluent fixed points is to be constructed, then let $D^{\prime \prime}=$ $D^{\prime} \cup\left(U\left(\left[a_{1}, b\right] \mid b \in B^{\prime \prime}\right)\right)$, and extend $f^{\prime}: D^{\prime} \rightarrow D^{\prime}$ to $f^{\prime \prime}: D^{\prime \prime} \rightarrow D^{\prime \prime}$ as follows: if $x \in D^{\prime}$, let $f^{\prime \prime}(x)=f^{\prime}(x)$. If $x \in D^{\prime \prime} \backslash D^{\prime}$, then $x=\lambda m+(1-\lambda) b$, for some $b \in B^{\prime \prime}$, with $m=\max \left(L(b) \cap D^{\prime}\right)$ and $0 \leqq \lambda<1$. Let $f^{\prime \prime}(x)=$ $\mu f^{\prime}(m)+(1-\mu) b$, where again $\mu=\lambda+\frac{1}{2} \lambda(1-\lambda)$. Then $f^{\prime \prime}$ has the fixed point set $A \cup B$, with the points of $A$ as noneffluent and the points of $B$ as effluent fixed points.

It remains to extend $f^{\prime \prime}: D^{\prime \prime} \rightarrow D^{\prime \prime}$ to $f: D \rightarrow D$. For this purpose, let $r: D \rightarrow D^{\prime \prime}$ be the retraction of Lemma $3.1, i: D^{\prime \prime} \rightarrow D$ be the injection, and define $f$ by $f=i \circ f^{\prime \prime} \circ r$. Clearly $f$ has the fixed point set Fix $f=A \cup B$. The points of $A$ are noneffluent, as $x \notin(a, f(x))$ for $x \in \operatorname{Bd} N(a)$, where $N(a)$ is any subdendrite of $D$ with $N(a) \cap$ Fix $f=$ $\{a\}$. In order to see that the points of $B$ are effluent take any $b \in B$, and any neighbourhood $U(b)$. Choose a neighbourhood $V(b) \subset U(b)$ with $\mathrm{Cl} V(b) \cap$ Fix $f=\{b\}$, and so that $\mathrm{Cl} V(b) \backslash\{b\}$ contains no branchpoints of $D^{\prime \prime}$. Let $W(b)$ be an open neighbourhood $W(b) \subset V(b)$ and so that Bd $W(b)$ consists of at most $o(b)$ points, and let $K(b)$ be the component of $W(b)$ which contains $b$. Finally put $N(b)=\mathrm{Cl} K(b)$. Then $N(b)$ is a neighbourhood of $b$ and is a subdendrite. As $N(b) \cap$ $D^{\prime \prime} \subset C l V(b) \cap D^{\prime \prime} \quad$ and $o(b)=o_{D^{\prime \prime}}(b)$, we have $N(b) \cap D^{\prime \prime}=$ $\cup\left(\left[b, x_{i}\right] \mid i=1,2, \cdots, o(b)\right)$, where $\left[b, x_{i}\right] \cap\left[b, x_{s}\right]=\{b\}$ for $i \neq j$. Hence $\left\{x_{i} \mid i=1,2, \cdots, o(b)\right\}$ is the boundary of $N(b) \cap D^{\prime \prime}$ in $D^{\prime \prime}$. Now $\mathrm{Bd}_{b^{\prime \prime}}\left(N(b) \cap D^{\prime \prime}\right) \subseteq \mathrm{Bd} W(b)$ and $\mathrm{Bd} W(b)$ consists of at most $o(b)$ points. So we see that $\mathrm{Bd} N(b)=\left\{x_{1} \mid i=1,2, \cdots, o(b)\right\}$. By construction of $f$ we have $x_{i} \in\left(b, f\left(x_{i}\right)\right)$ for all $i$, and therefore $N(b)$ satisfies the conditions in the definition of an effluent fixed point.

REMARK. In consequence of Lemma 5.1, we can replace the condition (iii) in Theorem 5.2 by 
(iii)

$$
\# A=1+\sum_{b \in B}(o(b)-1)
$$

But condition (ii) cannot be omitted even in the presence of condition (iii)'. Consider, e.g., the dendrite $D$ which consists of the points $0 \leqq x \leqq 4$ on the real line, let $A=\{0,1,4\}$ and $B=\{2,3\}$. Then $A$ and $B$ satisfy (i) and (iii)', but there is no selfmap of $D$ which has the points of $A$ as its noneffluent fixed points and the points of $B$ as its effluent fixed points.

\section{REFERENCES}

1. R. H. Bing, Partitioning continuous curves, Bull. Amer. Math. Soc., 58 (1952), 536-556.

2. R. F. Brown, The Lefschetz Fixed Point Theorem, Glenview, Ill., 1971.

3. C. E. Capei and W. L. Strother, Multi-valued functions and partial order, Portugal Math., 17 (1958), 41-47.

4. K. Kuratowski, Topology, vol. II, New Edition, New York and London, 1968.

5. L. Lum, A characterization of local connectivity in dendroids, Studies in Topology (Proc. Conf. Univ. North Carolina, Charlotte, N.C., 1974), New York, 1975, 331-338.

6. R. L. Plunkett, A fixed point theorem for continuous multi-valued transformations, Bull. Amer. Math. Soc., 7 (1956), 160-163.

7. H. Schirmer, Fixed point sets of polyhedra, Pacific J. Math., 52 (1974), 221-226.

8. - Mappings of polyhedra with prescribed fixed points and fixed point indices, Pacific J. Math., 63 (1976), 521-530.

9. L. E. Ward, Jr., Partially ordered topological spaces, Proc. Amer. Math. Soc., 5 (1954), 144-161.

10. —- A note on dendrites and trees, Proc. Amer. Math. Soc., 5 (1954), 992-994.

11. —_, A general fixed point theorem, Colloq. Math., 15 (1966), 243-251.

12. - Fixed point sets, Pacific J. Math., 47 (1973), 553-565.

13. G. T. Whyburn, Analytic Topology, Providence, R.I., 1942.

Received January 19, 1977 and in revised form May 27, 1977. This research was partially supported by the National Research Council of Canada (Grant A 7579).

Carleton University, Ottawa, Canada 


\section{PACIFIC JOURNAL OF MATHEMATICS}

\section{EDITORS}

RICHARD ARENS (Managing Editor)

University of California

Los Angeles, CA 90024

R. A. BeAumont

University of Washington

Seattle, WA 98105

C. C. MOORE

University of California

Berkeley, CA 94720
J. DUGUNDJI

Department of Mathematics

University of Southern California

Los Angeles, CA 90007

R. FINN AND J. MILGRAM

Stanford University

Stanford, CA 94305

\section{ASSOCIATE EDITORS}
E. F. BECKENBACH
B. H. NEUMANN
F. WOLF
K. YoshidA

\section{SUPPORTING INSTITUTIONS}

UNIVERSITY OF BRITISH COLUMBIA

UNIVERSITY OF SOUTHERN CALIFORNIA

CALIFORNIA INSTITUTE OF TECHNOLOGY

STANFORD UNIVERSITY

UNIVERSITY OF CALIFORNIA

UNIVERSITY OF HAWAII

MONTANA STATE UNIVERSITY

UNIVERSITY OF TOKYO

UNIVERSITY OF NEVADA

UNIVERSITY OF UTAH

NEW MEXICO STATE UNIVERSITY

OREGON STATE UNIVERSITY

UNIVERSITY OF OREGON

OSAKA UNIVERSITY

WASHINGTON STATE UNIVERSITY

UNIVERSITY OF WASHINGTON

AMERICAN MATHEMATICAL SOCIETY

The Supporting Institutions listed above contribute to the cost of publication of this Journal, but they are not owners or publishers and have no responsibility for its contents or policies.

Mathematical papers intended for publication in the Pacific Journal of Mathematics should be in typed form or offset-reproduced (not dittoed), double spaced with large margins. Underline Greek letters in red, German in green, and script in blue. The first paragraph or two must be capable of being used separately as a synopsis of the entire paper. Items of the bibliography should not be cited there unless absolutely necessary, in which case they must be identified by author and Journal, rather than by item number. Manuscripts, in duplicate, may be sent to any one of the four editors. Please classify according to the scheme of Math. Reviews, Index to Vol. 39. All other communications should be addressed to the managing editor, or Elaine Barth, University of California, Los Angeles, California, 90024.

100 reprints are provided free for each article, only if page charges have been substantially paid. Additional copies may be obtained at cost in multiples of 50 .

The Pacific Journal of Mathematics is issued monthly as of January 1966. Regular subscription rate: $\$ 72.00$ a year (6 Vols., 12 issues). Special rate: $\$ 36.00$ a year to individual members of supporting institutions.

Subscriptions, orders for numbers issued in the last three calendar years, and changes of address should be sent to Pacific Journal of Mathematics, 103 Highland Boulevard, Berkeley, California, 94708.

PUBLISHED BY PACIFIC JOURNAL OF MATHEMATICS, A NON-PROFIT CORPORATION

Printed at Jerusalem Academic Press, POB 2390, Jerusalem, Israel. 


\section{Pacific Journal of Mathematics}

\section{Vol. 75, No. $2 \quad$ October, 1978}

Susan Jane Zimmerman Andima and W. J. Thron, Order-induced

topological properties ................................... 297

Gregory Wade Bell, Cohomology of degree 1 and 2 of the Suzuki groups . . 319

Richard Body and Roy Rene Douglas, Rational homotopy and unique

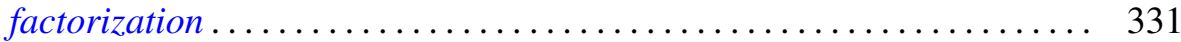

Frank Lewis Capobianco, Fixed sets of involutions ................. 339

L. Carlitz, Some theorems on generalized Dedekind-Rademacher sums .... 347

Mary Rodriguez Embry and Alan Leslie Lambert, The structure of a special class of weighted translation semigroups .....................

Steve Ferry, Strongly regular mappings with compact ANR fibers are

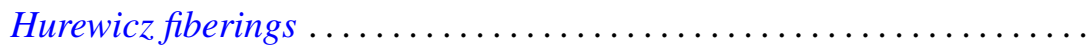

Ivan Filippenko and Marvin David Marcus, On the unitary invariance of the

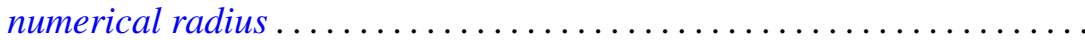

$\mathrm{H}$. Groemer, On the extension of additive functionals on classes of convex

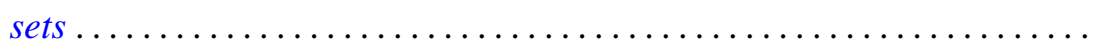

Rita Hall, On the cohomology of Kuga's fiber variety ............... 411

H. B. Hamilton, Congruences on $\mathrm{N}$-semigroups ................. 423

Manfred Herrmann and Rolf Schmidt, Regular sequences and lifting

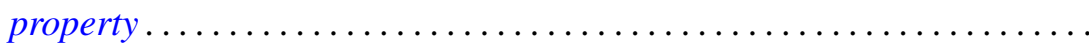

James Edgar Keesling, Decompositions of the Stone-Čech compactification which are shape equivalences .....................

Michael Jay Klass and Lawrence Edward Myers, On stopping rules and the expected supremum of $S_{n} / T_{n}$

Ronald Charles Linton, $\lambda$-large subgroups of $C_{\lambda}$-groups

William Owen Murray, IV and L. Bruce Treybig, Triangulations with the free cell property ............................

Louis Jackson Ratliff, Jr., Polynomial rings and $H_{i}$-local rings ...

Michael Rich, On alternate rings and their attached Jordan rings....

Gary Sampson and H. Tuy, Fourier transforms and their Lipschitz

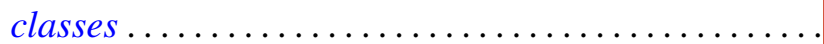

Helga Schirmer, Effluent and noneffluent fixed points on dendrites ...

Daniel Byron Shapiro, Intersections of the space of skew-symmetric maps

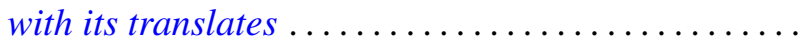

Edwin Spanier, Tautness for Alexander-Spanier cohomology ...

Alan Stein and Ivan Ernest Stux, A mean value theorem for binary digits ...

Franklin D. Tall, Normal subspaces of the density topology . .

William Yslas Vélez, Prime ideal decomposition in $F\left(\mu^{1 / p}\right) \ldots$ 